\title{
The Catsper channel and its roles in male fertility: a systematic review
}

\author{
Xiang-hong Sun ${ }^{1 *}$, Ying-ying Zhu², Lin Wang ${ }^{3}$, Hong-ling Liu' ${ }^{1}$, Yong Ling ${ }^{1}$, Zong-li Li ${ }^{1}$ and Li-bo Sun ${ }^{1}$
}

\begin{abstract}
The Catsper channel is a sperm-specific, $\mathrm{Ca}^{2+}$-permeable, pH-dependent, and low voltage-dependent channel that is essential for the hyperactivity of sperm flagellum, chemotaxis towards the egg, capacitation and acrosome reaction. All of these physiological events require calcium entry into sperm cells. Remarkably, Catsper genes are exclusively expressed in the testis during spermatogenesis, and are sensitive to ion channel-induced $\mathrm{pH}$ change, such as NHEs, $\mathrm{Ca}^{2+} \mathrm{ATPase}^{+} \mathrm{K}^{+}$ channel, $\mathrm{Hv} 1$ channel and $\mathrm{HCO}_{3}^{-}$transporters. Furthermore, the Catsper channel is regulated by some physiological stimulants, such as progesterone, cyclic nucleotides (e.g., cAMP, cGMP), zona pellucida (ZP) glycoproteins and bovine serum albumin (BSA). All of these factors normally stimulate $\mathrm{Ca}^{2+}$ entry into sperm through the Catsper channel. In addition, the Catsper channel may be a potential target for male infertility treatment or contraception. This review will focus on the structure, functions, regulation mechanisms and medicinal targets of the Catsper channel.
\end{abstract}

Keywords: Catsper channel, $\mathrm{Ca}^{2+}$ signal, Male infertility, Medicine

\section{Background}

Mature mammalian sperm execute many important physiological processes, such as sperm hyperactivation, chemotaxis towards the egg, capacitation and acrosome reaction, before even entering the female reproductive tract and contacting eggs for fertilization. Most studies show that all of physiological processes are closely related to the change of the calcium ion concentration $\left(\left[\mathrm{Ca}^{2+}\right] \mathrm{i}\right)[1,2]$ in sperm. There are two main sources for Calcium ions in sperm: (1) some of them are stored in a calcium pump located in the head of sperm, a redundant nuclear envelope in the same position as the IP3 receptor in the neck region, and (2) others are packed in the mitochondria of the midpiece $[3,4]$. Some processes in sperm depend on calcium ion channels opening in the cell membrane [5]. Several typical voltage-gated $\mathrm{Ca}^{2+}$ channels are located in testis, but most voltage-gated $\mathrm{Ca}^{2+}$ channels also take effect in other organs, such as brain and heart. It is only the Catsper ion channel that is exclusively expressed in spermatozoa. The whole-cell patch-clamp technique applied to mouse spermatozoa, showed direct electrophysiological characteristics of the

\footnotetext{
* Correspondence: m13210055172@163.com

Xiang-hong Sun and Ying-ying Zhu contributed equally to this work and are co-first author

'Department of pharmacy, the affiliated hospital of Qingdao University

Medical College, Qingdao 266555, China

Full list of author information is available at the end of the article
}

protein channels. $\mathrm{Ca}^{2+}$ currents were only detected in the Catsper channel. Catsper is a sperm- specific, $\mathrm{Ca}^{2+}$-permeable, $\mathrm{pH}$-sensitive and weakly voltage-dependent ion channel that is located in the membrane of the flagellar principal piece, The presence of an inactive Catsper protein in male mice induces infertility [6].

The Catsper channel is activated by intracellular alkaline $\mathrm{pH}$, as shown by whole-cell patch-clamp in 2006 [5]. This channel not only permits $\mathrm{Ca}^{2+}$ entry into spermatozoa under physiological conditions but also allows monovalent cations $\left(\mathrm{Na}^{+}, \mathrm{Cs}^{+}\right)$or a divalent cation $\left(\mathrm{Ba}^{2+}\right)$ to pass into spermatozoa if there is no extracellular $\mathrm{Ca}^{2+}$. The Catsper channel complex contains four $\alpha$ subunits (Catsper1-4 [5,7]) and at least three auxiliary subunits (Catsper $\beta$ (beta), Catsper $\gamma$ (gamma) and Catsper $\delta$ (delta) [8]). The first pore-forming Catsper subunit, Catsper1, was discovered in 2001 [9] and plays a vital role in spermatozoa motility. Catsper1 was detected during a search for sequence homologies to voltage-gated $\mathrm{Ca}^{2+}$ selective channels. Previous studies showed that there is no Catsper1 expression in Catsper2-lacking mice and also no Catsper2 expression in Catsper1-lacking mice [7]. These results indicated that stable expression of Catsper1 requires Catsper2, and vice versa. However, Catsper3 and Catsper4 proteins are expressed in Catsper1-deletion mice, suggesting that 
stable expressions of Catsper3 and Catsper4 does not depend on the expression of Catsper1 and Catsper2 [10]. Compared with unselected sperm, a much higher proportion of swim-up selected sperm expresses Catsper1 [11], suggesting that Catsper1 also plays a crucial role in sperm swimming. Further studies showed that Catsper null sperm cells could not be hyperactivated under physiological conditions [1, 12]. Interestingly, depolarization evoked an increase in intracellular $\mathrm{Ca}^{2+}\left(\left[\mathrm{Ca}^{2+}\right] \mathrm{i}\right)$ in WT sperm cells, but not in Catsper1 null spermatozoa [12]. The phenotypes of Catsper2 ${ }^{-1-}$, Catsper $^{-/-}$and Catsper $4^{-/-}$mice were indistinguishable from Catsper $1^{-/-}$mice, and their sperm also lacked the hyperactivate motility needed for fertilization [7, 13]. A whole sperm patch clamp of epididymal sperm showed that Catsper current is absent in Catsper $1^{-/-}$, Catsper $2^{-/-}$, $\mathrm{Catsper}^{-/-}$and Catsper $4^{-1-}$ mice $[6,7]$.

In recent years, the study on Catsper channel activators and inhibitors is surging. Wang HF found that Cadmium (Cd), a heavy metal and endocrine disruptor in environment, caused male infertility through reducing the expression of Catsper proteins [14]. Mannowetz N also conducted a study that pregnenolone sulfate as a Catsper activator. However, pristimerin and lupeol which both are steroid-like molecules can act as contraceptive compounds through affecting sperm hyperactivation [15]. Therefore, the Catsper ion channel has been implicated as a potential target for male infertility treatment and contraception. Nonetheless, the role of Catsper and its antagonist in these functions have been scarcely reported. Herein, this review focuses on the role of $\mathrm{Ca}^{2+}$ signaling in sperm function and the effects of medicines targeting the Catsper channel.

\section{The structure and localization of the Catsper channel complex}

The Catsper channel complex is encoded by at least seven genes. The structure and distribution of the Catsper subunits are all essential for the channel's function [7]. Catsper1-3 are specifically expressed in the testis, while Catsper4 is predominantly expressed in the testis, and there is also weak expression in placenta and lung tissues [16]. The Catsper family is confined to the principal piece of mature spermatozoa flagella in humans and animals. There is no organelle in the principal piece of spermatozoa, so researchers speculate that Catsper1-4 are localized to the principal piece's plasma membrane and involved in the regulation of flagella whipping.

Sequence homology of the four Catsper subunits in the transmembrane region is rare, and the variance ranges from 16 to $22 \%$ [5]. Catsper1 has a $21 \%$ homologous identity and $40 \%$ homologous similarity to Catsper2. Furthermore, the Catsper family also has relatively low sequence homologies among different species. The basic information of the seven Catsper genes in human and mouse are clearly shown in Table 1 and Table 2. The Catsper protein is widely expressed in mammals (mouse, rat, dog, human), sea squirt (Ciona intestinalis), and sea urchin (Strongylocentrotus purpuratus). Six transmembrane segments (S1-S6) are detected in separate pore-forming Catsper $\alpha$ subunits, forming two individual physiological active sites: the voltage sensor domain (S1-S4) and poreforming domain (S1-P loop- S6). S1-S4 are linked by a short cyclic structure, and it is noteworthy that there are several positively charged amino acid residues (lysine/arginine) in the fourth transmembrane segment (S4) functioning as a voltage sensor [8]. S5 and S6 are linked by a short and hydrophobic cyclic structure, and this region has a conserved homologous sequence $([\mathrm{T}] \times[\mathrm{D}] \times[\mathrm{W}])$, which selectively permits $\mathrm{Ca}^{2+}$ entry through the cell membrane. Catsper1 contains six neutral amino acid residues in voltage-sensitive channels, while Catsper2 contains four such residues and Catsper3 and Catsper4 contain only two. Four asubunits have coiled proteins in their $\mathrm{C}$ ends, forming a functional tetramer to constitute a whole channel. Catsper $\beta$, a recently discovered protein, contains two presumed transmembrane-spanning domains. Catsper $\beta$ is also the first identified auxiliary protein of the Catsper channel, expressed predominantly in the testis and the sperm tail [17].

\section{Catsper regulation and $\left[\mathrm{Ca}^{2+}\right]$ signaling}

Two $\mathrm{Ca}^{2+}$ channels regulate male fertility: (1) the Orail channel, which regulate store-operated calcium entry [18], and (2) the Catsper channel, which is the most extensively studied $\mathrm{Ca}^{2+}$ channels in mammalian sperm $[8,11]$. The sperm-specific Catsper channel controls the intracellular $\mathrm{Ca}^{2+}$ concentration $\left(\left[\mathrm{Ca}^{2+}\right] \mathrm{i}\right)$. Catsper1 and Catsper2 null mice exhibit lower amplitudes of flagellar bends compared to wild-type mice. In Catsper1 and Catsper2 null mice, the flagellar bend and amplitude are increased from abnormally low levels to normal prehyperactivated levels by increasing the spermospore $\left[\mathrm{Ca}^{2+}\right] \mathrm{i}$ [1]. In most mammals, sperm hyperactivated motility

Table 1 The essential information of Catsper subunits in human testes

\begin{tabular}{llll}
\hline Gene name & Chromosome (human) & exon & Amino acid \\
\hline Catsper1 & $11 \mathrm{q} 13.1$ & 12 & 780 \\
Catsper2 & $15 \mathrm{q} 15.3$ & 14 & 530 \\
Catsper3 & $5 \mathrm{q} 31.1$ & 8 & 344 \\
Catsper4 & $1 \mathrm{p} 36.11$ & 11 & 472 \\
Catsper $\beta$ & $14 \mathrm{q} 32.12$ & 27 & 1116 \\
Catspery & $19 \mathrm{q} 13.2$ & 36 & 1159 \\
Catsper8 & $19 \mathrm{p} 13.3$ & 25 & 798 \\
\hline
\end{tabular}


Table 2 The essential information of Catsper subunits in mice testes

\begin{tabular}{llll}
\hline Gene name & Chromosome (mouse) & exon & Amino acid \\
\hline Catsper1 & $19 \mathrm{~A}$ & 13 & 686 \\
Catsper2 & $2 \mathrm{E} 5$ & 16 & 588 \\
Catsper3 & $13 \mathrm{~B} 1$ & 9 & 395 \\
Catsper4 & $4 \mathrm{D} 3$ & 12 & 442 \\
Catsper $\beta$ & $12 \mathrm{E}$ & 27 & 1109 \\
Catspery & $7 \mathrm{~B} 1$ & 30 & 1145 \\
Catsper $\delta$ & $17 \mathrm{D}$ & 28 & 805 \\
\hline
\end{tabular}

depends on calcium influx into the sperm cytoplasm either from the extracellular space or released from intracellular organelles [3, 4]. Therefore, the Catsper channel controls, at least, the swimming behavior of sperm.

\section{$\mathrm{pH}$ regulates the Catsper ion channel}

The Catsper channel is a pH-sensitive ion channel, and a high $\mathrm{pH}$ level is necessary for sperm hyperactivation [19]. Thus, factors that regulate the acid-base properties also affect the degree of Catsper channel-opening in sperm. According to previous work, mouse sperm produces a $\mathrm{Ca}^{2+}$ increase in an artificially alkalinized intracellular environment [20]. Another studies also showed that progesterone, prostaglandins and ZP3 could induce capacitation and acrosome reaction in sperm by increasing $\left[\mathrm{Ca}^{2+}\right] \mathrm{i}$ $[21,22]$. However, later paper showed that the $\mathrm{Ca}^{2+}$ increases in sperm triggered by these bioactive molecules during acrosome reaction and capacitation are influenced by $\mathrm{pH}$ microenvironment in sperm [21]. Nevertheless, it is not completely clear how alkalinization influences the Catsper channel in sperm. $\mathrm{H}^{+}$is the major regulator of acid-base microenvironment, while $\mathrm{Na}^{+} / \mathrm{H}^{+}$exchangers (NHEs) and Voltage-gated $\mathrm{H}^{+}$channel1 (HV1) are $\mathrm{H}^{+}$-relative channels. NHEs import $\mathrm{Na}^{+}$into the plasma membrane and export $\mathrm{H}^{+}$out of the spermatozoa while HV1 removes intracellular $\mathrm{H}^{+}$to maintain the $\mathrm{pH}$ value $(\mathrm{pHi})$ balance in spermatozoa [23]. In addition, $\mathrm{Ca}^{2+}$ adenosine triphosphatase $\left(\mathrm{Ca}^{2+}\right.$ ATPase) pumps remove intracellular $\mathrm{Ca}^{2+}$ from spermatozoa while allowing $\mathrm{H}^{+}$to enter through the plasma membrane. Correspondently, the Catsper channel imports $\mathrm{Ca}^{2+}$ into spermatozoa to maintain $\mathrm{Ca}^{2+}$ homeostasis [24, 25]. Beyond that, the $\mathrm{Na}$, K-ATPase (NKA) and $\mathrm{Na}^{+} / \mathrm{Ca}^{2+}$ exchanger (NCX) also influence the ion milieu in human sperm [26]. In fact, high levels of intracellular $\mathrm{Ca}^{2+}$ and low levels of intracellular $\mathrm{H}^{+}$contribute to sperm hyperactivation. The relation of different ion channels with the Catsper channel is described in Fig. 1.

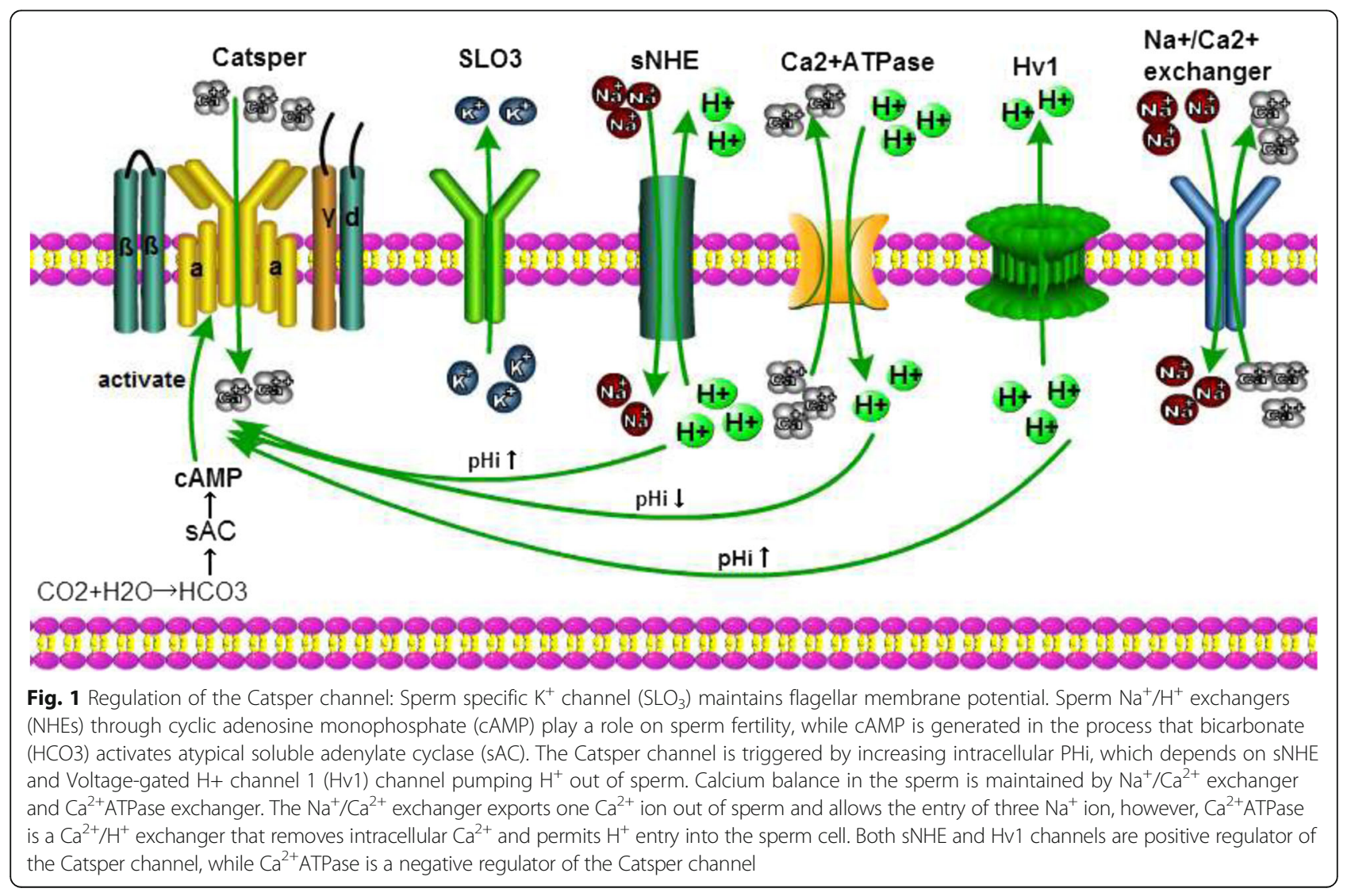




\section{$\mathrm{H}^{+}$channels}

(1) $\mathrm{Na}^{+} / \mathrm{H}^{+}$exchangers $\mathrm{Na}^{+} / \mathrm{H}^{+}$exchangers (NHEs) are responsible for the exchange of $\mathrm{Na}^{+}$and $\mathrm{H}^{+}$. Also they are integral membrane proteins that are widely distributed in all prokaryotic and eukaryotic organisms. NHEs includes 13 NHE isoforms encoded by SLC9 gene family, but only three subtypes (NHE1, NHE5 and sNHE) exist in sperm cells. Among them, sNHE is a crucial isoform for fertility [27]. Namely, knockouts of the NHE1 and NHE5 genes fail to generate infertility in mice, but sNHE-null or sNHE-disruption mice became infertile due to the loss of mobility and motility of spermatozoa [3]. Increased $\mathrm{pH}$ levels by providing ammonium chloride recovers partial fertility, but a cAMP analogue completely recoveres fertility [28]. Furthermore, sNHE includes a nucleotide-binding domain close to the protein's C-terminus [29]. Thus, sNHE isoform is necessary for fertility, and cyclic nucleotides may control the Catsper channel and increase pHi by activating sNHE. Moreover, sNHE helps maintain the alkaline environment, which allows the Catsper channel to further regulate hyperpolarization. Aside from maintaining the $\mathrm{pH}$ dynamic equilibrium in sperm, sNHE also regulates sperm maturity and promotes the absorption of salt and water in epithelial cell $[27,30]$. Interfering with the function of sNHE leads to infertility. Hence, targeting sNHE may be a promising strategy for developing novel contraception methods. Like the Catsper channel, sNHE is located in the principal piece of the sperm flagellum [28], suggesting that the Catsper channel can perceive $\mathrm{pHi}$ changes from sNHE to regulate the Catsper function.

(2) Hv1 channel Hv1 is another voltage-gated $\mathrm{H}^{+}$ channel. Similar to sNHE and the Catsper channel, Hv1 is also located in the principal piece of sperm flagella [31]. In terms of their functions, the major difference between Hv1 and sNHE is that Hv1 maintains intracellular alkalinization only by removing intracellular $\mathrm{H}^{+}$ from sperm [23]. Patch-clamp techniques have detected a negative current from the Hv1 channel during the physiological process of human sperm capacitation [32], which suggests that $\mathrm{Hv} 1$ is associated with sperm capacitation. Interestingly, this negative current is not detected from the mouse Hv1 channel [32]. In other words, there is no $\mathrm{H}^{+}$extrusion through $\mathrm{Hv1}$ in mouse sperm. We suspect that sNHE independently accomplishes acidbase regulation in mouse sperm. If so, knocking out the Hv1 gene in mouse should not affect fertility, but insufficient evidence has tested this hypothesis, and the actual mechanism of Hv1 in ouse needs further investigation.

\section{$K^{+}$channels}

As mentioned previously, sNHE-induced exchange of $\mathrm{Na}^{+}$and $\mathrm{H}^{+}$causes pHi increases, and both sNHE and
Catsper are voltage-dependent channels. In addition, cell membrane hyperpolarization correlates with capacitation. $\mathrm{K}^{+}$helps maintain the balance of the membrane potential in sperm. The $\mathrm{K}^{+}$channels that accomplish this balance are the SLO3 and Kir channels [33]. Among them, the SLO3 channel is a sperm-specific and $\mathrm{pH}$ sensitive $\mathrm{K}^{+}$channel. Similar to the Catsper channel, $\mathrm{SLO}_{3}$ is also strongly related to sperm hyperactivity and motility [34, 35]. Another crucial function of the $\mathrm{SLO}_{3}$ channel that affects the Catsper channel is to maintain the current balance of flagella in the spermospore [8]. The sperm resting transmembrane potential is approximately from -35 to $-45 \mathrm{mV}$, but when $\mathrm{K}^{+}$moves out of the cell and initiates hyperpolarization, the sperm transmembrane potential decreases to $-70 \mathrm{mV}$ [36]. A series of physiological processes are then triggered, including $\mathrm{Na}^{+} / \mathrm{H}^{+}$exchanger activation, sperm capacitation and sperm binding ZP3 [37].

\section{$\mathrm{Ca}^{2+}$ channels}

There are three $\mathrm{Ca}^{2+}$-related channels (Catsper channel, $\mathrm{Ca}^{2+}$ ATPase and $\mathrm{Na}^{+} / \mathrm{Ca}^{2+}$ exchanger) found in sperm. The Catsper channel is responsible for the entrance of $\mathrm{Ca}^{2+}$ into the spermospore, which promotes sperm motility. $\mathrm{Ca}^{2+}$ ATPase is a $\mathrm{Ca}^{2+} / \mathrm{H}^{+}$exchanger that removes intracellular $\mathrm{Ca}^{2+}$ and permits $\mathrm{H}^{+}$entry into the sperm cell different from the Catsper channel $[24,25]$. The $\mathrm{Ca}^{2+}$ ATPase can negatively regulate the Catsper channel and sperm fertilization. Furthermore, the $\mathrm{Na}^{+} / \mathrm{Ca}^{2+}$ exchanger exports one $\mathrm{Ca}^{2+}$ ion out of sperm and allows the entry of three $\mathrm{Na}^{+}$ion, which is essential to maintain the $\mathrm{Ca}^{2+}$ balance of the intracellular environment $[38,39]$.

\section{Bicarbonate $\left(\mathrm{HCO}_{3}^{-}\right)$transporters}

$\mathrm{HCO}_{3}^{-}$is indispensable for sperm capacitation [40], which is often considered as the beginning of early activation of sperm motility. For example, as mentioned above, mouse sperm treated with artificial alkalinization produces $\mathrm{Ca}^{2+}$ increasing [20]. The addition of $\mathrm{HCO}_{3}^{-}$ shows the same effect, while also increases the beat frequency of sperm [41]. These data suggest that transport of $\mathrm{HCO}_{3}^{-}$affects sperm motility by increasing the sperm pHi. Furthermore, $\mathrm{HCO}_{3}^{-}$activates atypical soluble adenylate cyclase (sAC), which increases the cAMP levels [42], and cAMP-mediated pathways, which increase the flagella beat frequency [43, 44] (cAMP-mediated pathways can activate the Catsper channel, as mentioned below). Thus, another mechanism that $\mathrm{HCO}_{3}^{-}$activates the Catsper channel is the promotion of $\mathrm{Ca}^{2+}$ increases, which may be through enhancing the generation of cAMP. In addition, CFTR is a $\mathrm{Cl}^{-}$and $\mathrm{HCO}_{3}^{-}$transmembrane transporter that is associated with human sperm capacitation [45]. CFTR controls many transport 
proteins by modulating the cAMP signaling pathway $[46,47]$. Remarkably, suppression of the CFTR transporter affects $\mathrm{HCO}_{3}^{-}$-induced cAMP increases, leading to decreased PKA activity. Moreover, decreasing CFTR activity also causes decreased tyrosine phosphorylation and decreased hyperactivated motility by modulating cAMP-downstream signaling cascades [48]. $\mathrm{HCO}_{3}^{-}$transporters are encoded by the SLC4, SLC26 and CFTR gene families in sperm, and these transporters constitute the main families of transmembrane proteins that are associated with the regulation of $\mathrm{pH}$ in mammalian cells. Western blotting, immunocytochemistry, qRT-PCR and immunoprecipitation data demonstrate that SLC26A3, SLC26A6, and SLC9A3R1 are detected in the midpieces of mouse sperm flagella. These proteins interact with each other to increase $\mathrm{pH}$, during capacitation and hyperpolarization [49]. On the other hand, $\mathrm{HCO}_{3}^{-}$and CFTR transporters are located in the midpieces of flagella, but they do not co-localize with the Catsper channel. We speculate that $\mathrm{HCO}_{3}^{-}$channels influence the open or closed state of the Catsper channel indirectly by affecting the acrosome reaction.

$\mathrm{HCO}_{3}^{-}$signals ealy activation of sperm motility. The generation of $\mathrm{HCO}_{3}^{-}$is accomplished by carbonic anhydrases (CAs) $\left(\mathrm{CO}_{2}+\mathrm{H}_{2} \mathrm{O} \mathrm{HCO}_{3}^{-}+\mathrm{H}^{+}\right)$. Therefore, CAs are essential for sperm during fertilization. Studies show that CAs function in three ways: (1) catalyzing the production of $\mathrm{HCO}_{3}^{-},(2)$ regulating the $\mathrm{pHi}$ in sperm, and (3) regulating the sperm acrosome reaction. CAII and CAIV are the core subunits that have catalytic activities, and knocking out either of them will decrease the sperm motility, speed of sperm motility and sperm beating frequency [44]. CAII is located in the principal piece of sperm where Catsper channel is also located. CAIV, on the other hand, is located within the plasma membrane of the entire sperm tail. CA inhibitors, such as ethoxyzolamide have been administered to human capacitated sperm and mouse capacitated sperm, and the results showed that the acrosome reaction increased in human capacitated sperm, but there were no increases in mouse capacitated sperm [50]. These results demonstrated that CAs exhibited different functions in human and mouse sperm. However, little is known about how CAs participate in sperm fertilization. CAs directly participate in maintaining the balance of ions during motility. For example, the flagellar beat frequency is increased when $\mathrm{CO}_{2}$ is administered to the spermatozoa, and this effect is suppressed by ethoxyzolamide. Compared with the CA activity in sperm from wild-type and $\mathrm{CAIV}^{-1-}$ mice, physiological role of CAIV is to provide sperm, with $\mathrm{HCO}_{3}^{-}$required for stimulating sAC [43]. In brief, CAs can affect the alkaline environment and $\mathrm{HCO}_{3}^{-}$concentrations in sperm. It is conceivable that CAs may affect the sperm acrosome raction by modulating the Catsper channel.

\section{Physiological stimuli that regulate the Catsper channel} How extracellular $\mathrm{Ca}^{2+}$ enters sperm is poorly understand, but several physiological stimulus associated with fertilization induce $\mathrm{Ca}^{2+}$ entry to increase $\left[\mathrm{Ca}^{2+}\right] \mathrm{i}$ through the Catsper channel. These stimuli include progesterone, cyclic nucleotides (e.g., cAMP, cGMP), ZP glycoproteins, BSA and alkaline depolarization [51]. Collectively, these elements induce a series of physiological events, including capacitation, acrosome reaction and fertilization.

\section{Cyclic nucleotide-induced $\mathrm{Ca}^{2+}$ entry}

The cAMP/PKA signaling pathway is used in mammals to regulate gene transcription. In fact, sperm capacitation is a cAMP-dependent process that up regulates the $\mathrm{Ca}^{2+}$ concentration and tyrosine phosphorylation levels [52]. Cyclic AMP is synthesized by many types of adenylyl cyclases (ACs) that are commonly divided into two groups: transmembrane adenylyl cyclases (tmACs) and sAC [19]. Importantly, sAC-mediated cAMP/PKA signal cascades are essential for sperm capacitation, because sAC-mutant or sAC-null mice can produce sperm, but sperm exhibit no forward motility, causing male infertility. Giving cAMP analogs to sAC-mutant or sAC-null mice completely restores the previously lost motility, but the sperm still exhibit no hyperactivity to fertilize eggs in vitro [53, 54]. Moreover, sAC not only plays a role in producting cAMP but also participates in other mechanisms involved in the fertilization process. Specifically, $\mathrm{sAC}$ have three roles in fertilization. First, sAC works as a $\mathrm{HCO}_{3}^{-}$sensor. The structural domains of sAC rearrange after being stimulating by $\mathrm{HCO}_{3}^{-}$, and $\mathrm{sAC}$ is activated by increasing cAMP levels in sperm $[54,55]$. The second function of $\mathrm{sAC}$ is to act as a $\mathrm{pH}$ sensor. Previous work showed that sAC regulate acid/base equilibrium in dogfish sperm [56], and its gene works as a monitor which reflects the concentration of $\mathrm{CO}_{2}$ and $\mathrm{HCO}_{3}^{-}$to keep an appropriate $\mathrm{pH}$ microenvironment [55]. The last function of $\mathrm{sAC}$ is to function as a $\mathrm{Ca}^{2+}$ sensor or calmodulin. As a substitute for $\mathrm{HCO}_{3}^{-}, \mathrm{Ca}^{2+}$ can stimulate a combination of sAC with ATP to produce cAMP $[57,58]$. Cyclic AMP is a second messenger molecule that is integral to many physiological processes, including sperm chemotaxis towards to the egg and capacitation. A study showed that extracellular cAMP/cGMP increases the $\mathrm{Ca}^{2+}$ concentration [59]. When providing 8 - $\mathrm{Br}$-cAMP/cGMP to the spermatophore or providing alkaline depolarization to activate the spermatophore, a Catsper-dependent increase in the intracellular $\mathrm{Ca}^{2+}$ concentration initiates at the principal piece and speads through the midpiece to finally reach the head. This process occurs in a matter of seconds. Furthermore, compared with wild-type sperm, Catsper1mutant sperm have lower intracellular ATP levels [59]. 
In addition, cGMP signalling function in marine invertebrates to transducer chemoattractants to increase in the $\left[\mathrm{Ca}^{2+}\right] \mathrm{i}$ in the flagellum, thereby increases swimming behavior during chemotaxis [60, 61]. All of these findings suggest that cyclic nucleotides induce $\mathrm{Ca}^{2+}$ influx in the principal piece, but there is no clear evidence showing that this cyclic nucleotide-mediated process directly participates in inducing $\left[\mathrm{Ca}^{2+}\right] \mathrm{i}$ increases. However, by using cells treated with 8-Br-cNMP, one study has demonstrated that a cyclic nucleotides modulates progesterone to ultimately increase $\left[\mathrm{Ca}^{2+}\right] \mathrm{i}[62]$.

\section{ZP-induced $\mathrm{Ca}^{2+}$ entry}

An oocytes in the female reproductive tract is coated with a protective exterior called ZP. The ZP protein surrounding the oocyte is important in the fertilization process, because only when sperm pass through the ZP protein to complete the acrosome reaction can participate in fertilization. In fact, ZP glycoproteins consist of three subunits in mice: ZP1, ZP2 and ZP3 [63, 64]. Whereas four subunits-ZP1, ZP2, ZP3 and ZP4 exist in human sperm [65]. How does contact between the sperm's acrosome and the egg's ZP cause an increase in $\left[\mathrm{Ca}^{2+}\right]$ i? In fact, the sperm needs capacity (which is achieved by $\left[\mathrm{Ca}^{2+}\right] \mathrm{i}$ increases) to pass through the $\mathrm{ZP}$ when the sperm contacts the egg. Exocytosis of secretory vesicles from the acrosome occur and ultimately, the acrosome reaction is accomplished [66-68]. Early research reported that in vivo experimental addition of ZP to capacitated sperm increases $\left[\mathrm{Ca}^{2+}\right] \mathrm{i}$, while addition of a $\mathrm{ZP}$ inhibitor (i.e., tyrphostin A48, pertussis toxin and 3quinuclidinyl benzilate) suppresses ZP-induced acrosome reactions and reduces the intracellular concentration of $\mathrm{Ca}^{2+}$ in mouse sperm [69-71]. These effects may be related to signal transduction through G-proteins [72]. Beta1, 4 galactosyltransferase-I (GalT-I) is a receptor of ZP3 that forms a complex of heterotrimeric G proteins. Sperm are still able to bind ZP3 in GalT-I-deleted mice, but sperm lose the ability to complete the acrosome reaction and cross the ZP [73]. Compared with sperm in mice that can complete the acrosome reaction, sperm in human not only require ZP3 binding but also require ZP4 binding [65]. In addition, mammalian transient receptor potential (Trp) proteins are $\mathrm{Ca}^{2+}$ channel receptors that are essential for regulating the entrance of $\mathrm{Ca}^{2+}$ into mouse sperm. Trp is activated by ZP3 activating G-protein and phospholipase $\mathrm{C}$ [74]. ZP-induced $\mathrm{Ca}^{2+}$ entry into sperm help generate the acrosome reaction and change sperm motility, and both of these functions are carried out with the help of Beta-defensin proteins expressed in the epididymis [75].

In recent years, studies have found that the Catsper channel plays a critical role in ZP-induced $\mathrm{Ca}^{2+}$ entry into mouse sperm. Using patch-clamp techniques, $\mathrm{Ca}^{2+}$ currents after $2 \mathrm{~min}$ of $\mathrm{ZP}$ stimulation were undetectable in Catsper1-null mouse sperm, showing that the Catsper channel is necessary for $\mathrm{ZP}$ to induce $\left[\mathrm{Ca}^{2+}\right] \mathrm{i}$ increases and suggesting that the $\mathrm{ZP}$-induced $\left[\mathrm{Ca}^{2+}\right] \mathrm{i}$ increases start from sperm tails and propagate toward the sperm heads [64].

\section{Progesterone induces $\mathrm{Ca}^{2+}$ increases}

Progesterone surrounds the egg in the female reproductive tract and is released by cumulus cells. Progesterone induces $\mathrm{Ca}^{2+}$ entry into sperm through the Catsper channel and thus promotes the acrosome reaction [76, 77]. How specifically progesterone regulates $\mathrm{Ca}^{2+}$ concentration has been deeply investigated. An increase of the $\left[\mathrm{Ca}^{2+}\right] \mathrm{i}$ was discovered when human spermatozoa were exposed to a concentration gradient of progesterone to simulate sperm to approach eggs. Direct addition of $\mathrm{Ca}^{2+}$ to medium failed to induce this process, but increases in the $\left[\mathrm{Ca}^{2+}\right] \mathrm{i}$ were blocked by adding a sarcoplasmic/endoplasmic inhibitor. These data suggest that progesterone-induced $\mathrm{Ca}^{2}$ ${ }^{+}$influx is mediated by the release of stored $\mathrm{Ca}^{2+}$ in sperm, and thus may influence sperm behavior [78]. One mechanism of the Catsper channel to increase the $\left[\mathrm{Ca}^{2+}\right] \mathrm{i}$ is through releasing stored $\mathrm{Ca}^{2+}$, and we hypothesize that progesterone-induced $\mathrm{Ca}^{2+}$ influx may be mediated by the Catsper channel. One study recorded the Catsper currents from human epididymal and testicular spermatozoa, and the results showed that the Catsper channel is sensitive to progesterone early in sperm development and this sensitivity increases gradually to a peak when spermatozoa are ultimately ejaculated [79]. Protein kinases and phosphatases participate in progesterone-induced $\mathrm{Ca}^{2+}$ increases: the addition of PKA inhibitor or protein tyrosine phosphatase inhibitors reduced progesterone-induced $\mathrm{Ca}^{2+}$ influx and progesterone-induced acrosome rections [77]. In 2010, two research groups proposed that as a progesterone receptor in fish sperm, the Catsper channel is functioning to increase intracellular $\mathrm{Ca}^{2+}$ concentrations [80, 81]. In addition, in 2011, a study showed that progesterone is a steroid hormone that activates the Catsper channel in human sperm by regulating Catsper gene expression through a well-characterized progesterone nuclear receptor. Additionally, the Catsper protein is a non-genomic progesterone receptor [82], and $\mathrm{Ca}^{2+}$ influx is stimulated by alkaline $\mathrm{pH}$ and progesterone, but blocked by the Catsper inhibitors NNC55-0396 and mibefradil [83].

\section{Other stimuli induce $\mathrm{Ca}^{2+}$ entry through the Catsper channel}

One additional stimuli that promotes $\mathrm{Ca}^{2+}$ entry into sperm via the Catsper channel is BSA. BSA plays a role in sperm capacitation in several mammals. BSA also induces an increase in the intracellular $\mathrm{Ca}^{2+}$ concentration, but this effect is absent in Catsper1-knockout 
sperm. Addition to a EGFP-Catsper1 fusion protein recovered BSA-induced intracellular $\mathrm{Ca}^{2+}$ concentration increases [84]. The changes in calcium concentrations observed with BSA propagated from the principal piece to the mid-piece and, ultimately, the head within a few seconds [59].

\section{Functions of the Catsper protein channel}

Catsper was identified in mouse sperm as a putative $\mathrm{Ca}^{2+}$ channel in 2001 [9]. The Catsper channel is essential for male fertilization, especially for some physical processes, such as sperm hypermotility, egg penetration and the acrosome reaction. As such, the Catsper channel may be a target for male contraception. The structure of sperm and oocyte are showed in Fig. 2.

\section{Catsper and male fertility}

A plethora of research has proven that the Catsper channel is essential for both human and mouse fertility. A routine semen analysis in two consanguineous families that showed autosomal-recessive male infertility discovered that both families suffer from Catsper1 gene abnormalities. In different patients, asthenoteratozoospermia was diagnosed and found to lack the Catsper2 gene $[85,86]$. Furthermore, studies have also shown that

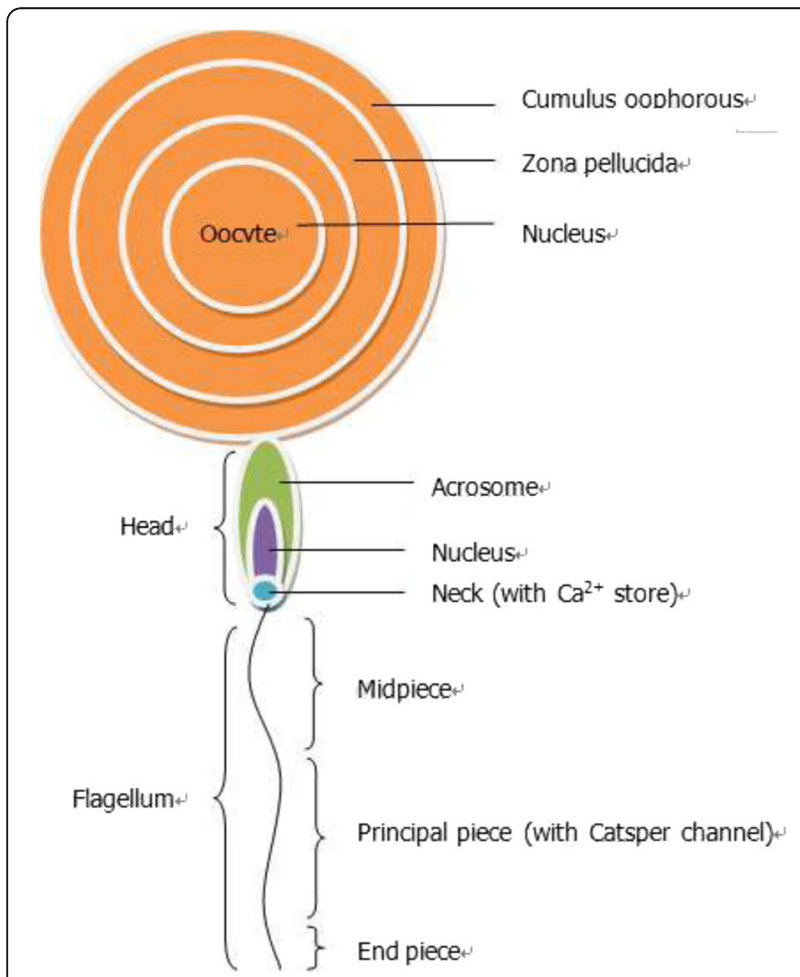

Fig. 2 The structure of sperm and oocyte: The sperm is constituted of acrosome, nucleus, neck and flagellum. The oocyte is constituted of cumulus oophorous, ZP and nucleus. Acrosome reaction is the start of fertilization all four Catsper subunits (Catsper1-4) are integral for sperm hyperactivated motility and male fertility, but lack of Catsper3 or Catsper4 has not influence spermatogenesis or initial motility of sperm [7]. Moreover, the number of sperm with progressive motility and progesteroneinduced acrosome reactions is significantly lower in Catsper1-suppressived groups than that in normal groups [11]. Normal expression of the Catsper channel is associated with progressive motility and acrosome reaction, abnormal channel expression may be involved in the pathogenesis of asthenozoospermia. Specifically, disruption of the sNHE or Catsper2 genes in mice cause male infertility, with findings of immotile spermatozoa and failed hyperactivated motility, but no other apparent abnormalities [3]. All of these results suggest that Catsper1 and Catsper2 are essential for normal male fertility in humans or mice. Interestingly, Catsper3 and 4 play an important role in the acrosome reaction and male fertility [87], which occurs not only in the testes but also in other tissues. On the other hand, Catsper1 and Catsper2 are only detected in mammalian testes. Collectively these results suggest that Catsper1 and Catsper2 are highly specialized flagellar proteins that are more important than Catsper3 and Catsper4 in sperm. There is no report showing that mutations of Catpser $\beta$ or Catspery lead to infertility [88]. However, these mutations show an abnormal detection of $\mathrm{Ca}^{2+}$ currents and hyperactivated motility in spermatozoa. Finally, Catsper $\delta$ knockout mice are infertile [89].

\section{Catsper and contraception}

The Catsper channel is a polymodal chemosensor that may be a target for contraception. Li aimed to explore the contraceptive potential of the Catsper1 transmembrane domains and pore region in vitro in human and mouse sperm. A significant decrease in sperm progressive motility was noticed after incubating cells with anti-Catsper1 lgG [90], demonstrating that Catsper1 may be a potential target for immunocontraception and that the antibody is a useful tool to study the function of ion channels in sperm. Later, $\mathrm{Li}$ additionally evaluated the contraceptive abilities of two Bcell epitopes in the transmembrane domains and pore region of Catsper1 in mice. Two predicted B-cell epitopes of the extracellular part of the transmembrane domains and pore region of Catsper1 were synthesized to immunize male mice. A significant reduction of fertility was observed in mating trials, with no evident systemic illnesses or abnormal mating behaviors suggesting that Catsper members may be effective and viable targets for immunocontraception. These two epitopes in Catsper1 share high identity between mouse and human and thus may be effective for regulating fertility in humans [91]. Novel drugs targeting the Catsper channel are warranted to study their potential roles in reversibly acting as male contraception. 
Pharmacological targeting of the Catsper channel With more advanced emerging methods that employ animal or cell models, more studies testing drugs that act as Catsper agonists or inhibitors are needed to test the channel's potential role as a target for infertility treatment and male contraception. The drugs that target the Catsper channel can be divided into three groups according to their pharmacological actions. Few of them are approved by the US Food and Drug Administration (FDA) for clinical therapies. Most of these drugs are at the stage of laboratory experiments, while a large portion of them are plant extracts.

\section{Anticholinesterase drugs}

In early studies, the organochlorine compounds of semen in infertile men have been analyzed, and the results showed that the concentrations of dichlorodiphenyltrichloroethane (DDT) and its metabolites, such as hexachlorocyclohexane $(\mathrm{HCH}), \mathrm{P}^{\mathrm{P}} \mathrm{P}^{\prime}$-dichlorodiphenyldichloroethylene (pp'-DDE) and pp'-DDD, are higher in the semen of infertile men than that in fertile men. It has been demonstrated that anticholinesterases affect male fertility by damaging the prostate [92-95]. Another in-vitro experiment, however, found that P,P'-DDE was a Catsper agonist that stimulated Catsper channel opening and caused $\mathrm{Ca}^{2+}$ influx into sperm [96]. In other words, P,P'-DDE may improve sperm fertility. Thus, P,P,DDE is a controversial compound compounds in sperm fertility.

\section{$\mathrm{Ca}^{2+}$ channel blockers}

Compounds such as HC-056456, NNC55-0396, nifedipine, nimodipine, quinindium, clofilium, theophylline and ketamine are all $\mathrm{Ca}^{2+}$ channel blockers. Among them, HC-056456 is a novel $\mathrm{Ca}^{2+}$ channel blocker, which is reported to be a unique compound that selectively targets the Catsper channel. Whole cell patch-clamp recordings showed a lower Catsper current in HC-056456 treated sperm than in untreated sperm, and HC-056456 reversibly prevented the development of hyperactivated motility of capacitated sperm [97]. This effect is similar to the findings of Catsper-null sperm, thus, HC-056456 is a promising compound that should be studied further as a male contraceptive. Both nifedipine and nimodipine are L-type $\mathrm{Ca}^{2+}$ channel blockers $[98,99]$, and $20 \mathrm{mg} / \mathrm{L}$ of both compounds could induce male infertility. Nifedipine also targets the Catsper chanel and prevents $\mathrm{Ca}^{2+}$ influx into sperm, which consequently alters the cholestenone content in sperm membranes leading to membrane disruption [10]. NNC55-0396 and mibefradil are two T-type $\mathrm{Ca}^{2+}$ channel blockers that are odorants and suppress $\mathrm{Ca}^{2+}$ signals under standard (physiological) conditions [100]. NNC (10 mM) and Mib (30 mM) significantly decrease the percentage of sperm with progressive motility and other kinematic parameters, but the compounds do not affect the percentage of hyperactivated sperm [11]. Other odorants, such as cyclamen and helional, evoke $\mathrm{Ca}^{2+}$ signals [51], these compounds are extracted from plants and bacteria and may act as potent molecules to treat Catsper-related male infertility. In addition, whole cell patch-clamp recordings of human sperm Catsper ion channels showed that quinidine reversibly blocks $\mathrm{Ca}^{2+}$ currents in the Catsper channel. Clofilium, on the other hand, causes irreversible blockade of Catsper-mediated $\mathrm{Ca}^{2+}$ currents [100-102]. A recent study showed that ketamine affects human sperm functions as well by inhibiting sperm total motility and progressive motility via decreased sperm $\mathrm{Ca}^{2+}$ influx [103].

\section{Other therapeutics that affect the Catsper channel}

The mechanisms of some drugs are not clearly understand. For example, emodin inhibits human sperm function by reducing the sperm $\left[\mathrm{Ca}^{2+}\right] \mathrm{i}$ and tyrosine phosphorylation [104]. Furthermore, some herbal plants, such as Trigonellae Semen (TS) and Panax ginseng, generate hyperactivity of sperm by regulating the expression of the Catsper gene. Extracting pure compounds from both TS and Panax ginseng may cure oligoasthenospermia $[105,106]$. In addition, matrine significantly inhibits sperm total motility, capacitation linear velocity and the progesterone-induced acrosome reaction by stimulating the Catsper channel [107], thus, matrine could be an potent drug to treat male contraception, While further clinic trails and systematic evaluations of these molecules are warranted.

At this point, drugs targeting on the Catsper channel remains in preclinical research stages. More intensive study of the Catsper channel as a target for treating is needed.

\section{The effects of the Catsper gene promoter on its transcription}

The regulation of the Catsper channel has been systematically studied at the protein level, but few researches reveal the effects of the Catsper promoter regions on the transcription of the Catsper gene. One group studied the promoter regions of the Catsper channel in human and murine sperm. Electrophoretic Mobility Shift Assays (EMSA) and DNA footprinting techniques were used to analyze the Catsper gene characteristics. There is a retardant when the Catsper gene and nucleoprotein were incubated together in vitro, suggesting some nucleoproteins have combined with Catsper gene promoter. Moreover, three transcription factor binding sites for SRY, SOX and CREB have been found in the Catsper promoter regions [108]. This group also demonstrated that the transcription factors SOX5 and SOX9 regulate the expression of the Catsper1 gene [109]. The role of the other transcription factors are still unknown. In addition, 
a toxicology study showed that cyclophosphamide (CP), a antineoplastic drug, could cause male infertility and lead to a significant reduction of the CREM transcription factor within the CREB transcription factor family [110]. The concentrations of CREM in spermatophores is 100 times greater than in other organizations of cells [111]. CREM-knockout mice also induce infertility. Thus, CREM is an important transcription factor in regulating the Catsper channel.

\section{Conclusion and perspective}

The Catsper channel plays a critical role in male fertility by controlling $\mathrm{Ca}^{2+}$ influx into spermatozoa. Many studies revealed protein and hormone based regulatory mechanism of the Catsper channel. In this review, we discussed how ion channels and stimulants influence the Catsper channel and induce $\mathrm{Ca}^{2+}$ entry into sperm. Thus far, a variety of $\mathrm{Ca}^{2+}$ channels have been found, and these channels (i.e., high voltage-gated $\mathrm{Ca}^{2+}$ channel (Cav), cyclic nucleotide-gated $\mathrm{Ca}^{2+}$ channel (CNG) and the TRP channel) are distributed in different subregions of sperm. In addition, pharmacological evidence shows that there are $\mathrm{N}$-type, $\mathrm{R}$-type and $\mathrm{T}$-type voltage-gated $\mathrm{Ca}^{2+}$ channels in sperm cells, but these channels do not directly affect sperm movement and fertility [112]. Only the Catsper channel directly modulates the physiological processes of sperm hyperactivation, sperm capacitation, chemotaxis towards to the egg and the acrosome reaction [8]. Many questions remain unanswered, though. Catsper is a $\mathrm{pH}$-sensitive ion channel. Some ion channels or enzymes (i.e., sNHE, CAs and $\mathrm{HCO}_{3}^{-}$transporter) alter sperm $\mathrm{pH}$ by changing the concentration of $\mathrm{H}^{+}$ ions. We know that all these biological molecules affect the opening degree of the Catsper channel, but are all of them essential for the Catsper channel? If one of them is mutated or deleted, can the Catsper channel continue to function normally? The mechanism of how these biological molecules interact with each other is not very clear. Furthermore, the sperm sNHE exchanger also acts via cAMP, however, the mechanism has not been clearly demonstrated. On the other hand, cAMP, ZP, progesterone and BSA are proteins that promote $\mathrm{Ca}^{2+}$ entry into Catsper channel. These compounds promote capacitation, the acrosome reaction, sperm maturation and sperm combining with an egg. What prevents activation of Catsper1 and Catsper2 in heterologous systems, and what other cell conditions are required to achieve activation? Other than the ZP, progesteronel, nucleotides and BSA, what other elements can increase $\mathrm{Ca}^{2+}$ influx through the Catsper channel? All of these questions need further exploration.

In addition, Catsper channel controls $\mathrm{Ca}^{2+}$ flux into spermatozoa and adjusts to hyperactivation of sperm. Thus, Catsper channel has been implicated as a potential target for contraception, and there are a lot of potent Catsper channel blockers for contraception, such as HC056456, NNC55-0396 and so on. Moreover, in 2017, Zou also found that diethylstilbestrol (DES), a Catsper activator, facilitated $\mathrm{Ca}^{2+}$ flux into human spermatozoa by Catsper channel [113]. Even so, the studies on Catsper agonists is still very rare. So it is necessary to study the mechanism of Catsper channel to research Catsper-targeted drugs treating male infertility.

The Catsper channel is a unique $\mathrm{Ca}^{2+}$ channel that is only detected in testes, so we speculate that Catsper may have unique factors or protein-protein interactions that contribute to the unique properties and regulation of the Catsper channel. In fact, there are few studies about the channel's promoter regions, and only four binding sequences on the Catsper1 gene promoter have been discovered [73]. No studies have described the remainder of the Catsper gene, so searching for new Catsper promoter binding sequences is important for finding potential molecular targets that could be used to treat male infertility. In addition, at the protein level, there are only two transcription factors (SOX5 and SOX9) that have been shown to regulate the transcription of the Catsper1 gene [109]. The SOX protein is a ubiquitously utilized transcription factor in many organisms, so the existence of another more specific protein that acts only on the Catsper promoter sequence may be an important area for future studies.

\section{Acknowledgments \\ We wish to acknowledge the following for support with this study: technology bureau of Qingdao; Qingdao University and the affiliated hospital of Qingdao University Medical College. \\ Funding \\ Science-technology Support Projects of Qingdao(2012-1-3-2-(7)-nsh).}

Availability of data and materials

Not applicable (a review article).

\section{Authors' contributions}

All authors contributed to the concepts of the manuscript; YYZ. wrote the first draft of the manuscript. XHS. made a modification of the manuscript. Other authors gave a further modification of the manuscript. All authors contributed to the writing process of the manuscript and approved the final version.

Ethics approval and consent to participate

Not applicable (a review article).

Consent for publication

NA.

Competing interests

The authors declare that they have no competing interests.

\section{Publisher's Note}

Springer Nature remains neutral with regard to jurisdictional claims in published maps and institutional affiliations.

\section{Author details}

${ }^{1}$ Department of pharmacy, the affiliated hospital of Qingdao University Medical College, Qingdao 266555, China. ²Department of pharmacy, College 
of pharmacy of Qingdao University, Qingdao, China. ${ }^{3}$ Department of clinical laboratory, the affiliated hospital of Qingdao University Medical College, Qingdao, China.

Received: 23 April 2017 Accepted: 3 August 2017

Published online: 15 August 2017

\section{References}

1. Marquez B, Ignotz G, Suarez SS. Contributions of extracellular and intracellular ca2+ to regulation of sperm motility: release of intracellular stores can hyperactivate Catsper1 and Catsper 2 null sperm. Dev Biol. 2007;303(1):214-21.

2. Chung JJ, Shim SH, Everley RA, Gygi SP, Zhuang X, Clapham DE. Structurally distinct $\mathrm{Ca}(2+)$ signaling domains of sperm flagella orchestrate tyrosine phosphorylation and motility. Cell. 2014;157(4):808-22.

3. Quill TA, Wang D, Garbers DL. Insights into sperm cell motility signaling through snhe and the Catspers. Mol Cell Endocrinol. 2006;250(1-2):84-92.

4. Costello S, Michelangeli F, Nash K, Lefievre L, Morris J, Machado-Oliveira G, Barratt C, Kirkman-Brown J, Publicover S. Ca2+-stores in sperm: their identities and functions. Reproduction. 2009;138(3):425-37.

5. Navarro B, Kirichok Y, Chung JJ, Clapham DE. Ion channels that control fertility in mammalian spermatozoa. Int J Dev Biol. 2008:52(5-6):607-13.

6. Kirichok Y, Navarro B, Clapham DE. Whole-cell patch-clamp measurements of spermatozoa reveal an alkaline-activated ca2+ channel. Nature. 2006; 439(7077):737-40

7. Qi H, Moran MM, Navarro B, Chong JA, Krapivinsky G, Krapivinsky L, Kirichok Y, Ramsey IS, Quill TA, Clapham DE. All four Catsper ion channel proteins are required for male fertility and sperm cell hyperactivated motility. Proc Natl Acad Sci U S A. 2007;104(4):1219-23.

8. Singh AP, Rajender S. Catsper channel, sperm function and male fertility. Reprod BioMed Online. 2015;30(1):28-38.

9. Ren D, Navarro B, Perez G, Jackson AC, Hsu S, Shi Q, Tilly JL, Clapham DE. A sperm ion channel required for sperm motility and male fertility. Nature. 2001;413(6856):603-9.

10. Babcock DF. Wrath of the wraiths of Catsper3 and Catsper4. Proc Natl Acad Sci U S A. 2007;104(4):1107-8

11. Tamburrino L, Marchiani S, Minetti F, Forti G, Muratori M, Baldi E. The Catsper calcium channel in human sperm: relation with motility and involvement in progesterone-induced acrosome reaction. Hum Reprod. 2014;29(3):418-28.

12. Carlson AE, Westenbroek RE, Quill T, Ren D, Clapham DE, Hille B, Garbers DL, Babcock DF. Catsper1 required for evoked ca2+ entry and control of flagellar function in sperm. Proc Natl Acad Sci U S A. 2003;100(25):14864-8

13. Quill TA, Sugden SA, Rossi KL, Doolittle LK, Hammer RE, Garbers DL. Hyperactivated sperm motility driven by Catsper2 is required for fertilization. Proc Natl Acad Sci U S A. 2003;100(25):14869-74.

14. Wang HF, Chang M, Peng TT, Yang Y, Li N, Luo T, Cheng YM, Zhou MZ, Zeng $\mathrm{XH}$, Zheng LP. Exposure to cadmium impairs sperm functions by reducing Catsper in mice. Cell Physiol Biochem. 2017:42(1):44-54.

15. Mannowetz N, Miller MR, Lishko PV. Regulation of the sperm calcium channel Catsper by endogenous steroids and plant triterpenoids. Proc Natl Acad Sci USA. 2017;114(22):5743-8

16. Lobley A, Pierron V, Reynolds L, Allen L, Michalovich D. Identification of human and mouse Catsper3 and Catsper4 genes: characterisation of a common interaction domain and evidence for expression in testis. Reprod Biol Endocrinol. 2003;1:53.

17. Liu J, Xia J, Cho KH, Clapham DE, Ren D. Catsperbeta, a novel transmembrane protein in the Catsper channel complex. J Biol Chem. 2007;282(26):18945-52.

18. Davis FM, Goulding EH, D'Agostin DM, Janardhan KS, Cummings CA, Bird GS, Eddy EM, Putney JW. Male infertility in mice lacking the store-operated ca(2+) channel orai1. Cell Calcium. 2016;59(4):189-97.

19. Nishigaki T, Jose $\mathrm{O}$, Gonzalez-Cota AL, Romero F, Trevino CL, Darszon A. Intracellular ph in sperm physiology. Biochem Biophys Res Commun. 2014:450(3):1149-58

20. Santi CM, Santos T, Hernandez-Cruz A, Darszon A. Properties of a novel phdependent ca2+ permeation pathway present in male germ cells with possible roles in spermatogenesis and mature sperm function. J Gen Physiol. 1998;112(1):33-53.

21. Arnoult C, Zeng Y, Florman HM. Zp3-dependent activation of sperm cation channels regulates acrosomal secretion during mammalian fertilization. J Cell Biol. 1996;134(3):637-45.
22. Sagare-Patil V, Vernekar M, Galvankar M, Modi D. Progesterone utilizes the pi3kakt pathway in human spermatozoa to regulate motility and hyperactivation but not acrosome reaction. Mol Cell Endocrinol. 2013;374(1-2):82-91.

23. Florman HM, Jungnickel MK, Sutton KA. Shedding light on sperm phertility. Cell. 2010;140(3):310-2.

24. Okunade GW, Miller ML, Pyne GJ, Sutliff RL, O'Connor KT, Neumann JC, Andringa A, Miller DA, Prasad V, Doetschman T, Paul RJ, Shull GE. Targeted ablation of plasma membrane ca2+-atpase (pmca) 1 and 4 indicates a major housekeeping function for pmca1 and a critical role in hyperactivated sperm motility and male fertility for pmca4. J Biol Chem. 2004;279(32):33742-50.

25. Schuh K, Cartwright EJ, Jankevics E, Bundschu K, Liebermann J, Williams JC, Armesilla AL, Emerson M, Oceandy D, Knobeloch KP, Neyses L. Plasma membrane ca2+ atpase 4 is required for sperm motility and male fertility. J Biol Chem. 2004;279(27):28220-6

26. Peralta-Arias RD, Vivenes CY, Camejo MI, Pinero S, Proverbio T, Martinez E, Marin R, Proverbio F. Atpases, ion exchangers and human sperm motility. Reproduction. 2015;149(5):475-84.

27. Fuster DG, Alexander RT. Traditional and emerging roles for the slc9 na+/h+ exchangers. Pflugers Arch. 2014;466(1):61-76.

28. Wang D, King SM, Quill TA, Doolittle LK, Garbers DL. A new sperm-specific nat/h+ exchanger required for sperm motility and fertility. Nat Cell Biol. 2003;5(12):1117-22.

29. Van Petegem F, Chatelain FC, Minor DL Jr. Insights into voltage-gated calcium channel regulation from the structure of the cav1.2 iq domain-ca2+/calmodulin complex. Nat Struct Mol Biol. 2005;12(12):1108-15.

30. Martins AD, Bernardino RL, Neuhaus-Oliveira A, Sousa M, Sa R, Alves MG, Oliveira PF. Physiology of nat/h+ exchangers in the male reproductive tract: relevance for male fertility. Biol Reprod. 2014;91(1):11.

31. Lishko PV, Kirichok Y. The role of hv1 and Catsper channels in sperm activation. J Physiol. 2010;588(Pt 23):4667-72.

32. Lishko PV, Botchkina IL, Fedorenko A, Kirichok Y. Acid extrusion from human spermatozoa is mediated by flagellar voltage-gated proton channel. Cell. 2010;140(3):327-37.

33. Acevedo JJ, Mendoza-Lujambio I, de la Vega-Beltran JL, Trevino CL, Felix R, Darszon A. Katp channels in mouse spermatogenic cells and sperm, and their role in capacitation. Dev Biol. 2006:289(2):395-405.

34. Escoffier J, Navarrete F, Haddad D, Santi CM, Darszon A, Visconti PE. Flow cytometry analysis reveals that only a subpopulation of mouse sperm undergoes hyperpolarization during capacitation. Biol Reprod. 2015;92(5):121.

35. Vilchez MC, Morini M, Penaranda DS, Gallego V, Asturiano JF, Perez L. Role of potassium and ph on the initiation of sperm motility in the european eel, comparative biochemistry and physiology. A Mol Integr Physiol. 2016; 203:210-9.

36. Munoz-Garay C, De la Vega-Beltran JL, Delgado R, Labarca P, Felix R, Darszon A Inwardly rectifying $\mathrm{k}(+)$ channels in spermatogenic cells: functional expression and implication in sperm capacitation. Dev Biol. 2001;234(1):261-74.

37. Arnoult C, Kazam IG, Visconti PE, Kopf GS, Villaz M, Florman HM. Control of the low voltage-activated calcium channel of mouse sperm by egg zp3 and by membrane hyperpolarization during capacitation. Proc Natl Acad Sci USA. 1999;96(12):6757-62.

38. Krasznai Z, Krasznai ZT, Morisawa M, Bazsane ZK, Hernadi Z, Fazekas Z, Tron L, Goda K, Marian T. Role of the na+/ca2+ exchanger in calcium homeostasis and human sperm motility regulation. Cell Motil Cytoskeleton. 2006;63(2):66-76.

39. Wennemuth G, Babcock DF, Hille B. Calcium clearance mechanisms of mouse sperm. J Gen Physiol. 2003:122(1):115-28.

40. Zhou Y, Ru Y, Shi H, Wang Y, Wu B, Upur H, Zhang Y. Cholecystokinin receptors regulate sperm protein tyrosine phosphorylation via uptake of hco3. Reproduction. 2015;150(4):257-68.

41. Carlson AE, Hille B, Babcock DF. External ca2+ acts upstream of adenylyl cyclase sacy in the bicarbonate signaled activation of sperm motility. Dev Biol. 2007;312(1):183-92.

42. Hereng TH, Elgstoen KB, Eide L, Rosendal KR, Skalhegg BS. Serum albumin and hco3- regulate separate pools of atp in human spermatozoa. Hum Reprod. 2014:29(5):918-30.

43. Wandernoth PM, Raubuch M, Mannowetz N, Becker HM, Deitmer JW, Sly WS, Wennemuth G. Role of carbonic anhydrase iv in the bicarbonate-mediated activation of murine and human sperm. PLoS One. 2010;5(11):e15061.

44. Wandernoth PM, Mannowetz N, Szczyrba J, Grannemann L, Wolf A Becker HM, Sly WS, Wennemuth G. Normal fertility requires the expression of carbonic anhydrases ii and iv in sperm. J Biol Chem. 2015;290(49):29202-16. 
45. Romero MF, Chen AP, Parker MD, Boron WF. The slc4 family of bicarbonate (hco(3)(-)) transporters. Mol Asp Med. 2013;34(2-3):159-82

46. Chavez JC, Hernandez-Gonzalez EO, Wertheimer E, Visconti PE, Darszon A, Trevino CL. Participation of the $\mathrm{Cl}-/ \mathrm{hco}(3)$ - exchangers slc26a3 and slc26a6, the $\mathrm{cl}$ - channel $\mathrm{cftr}$, and the regulatory factor slc9a3r1 in mouse sperm capacitation. Biol Reprod. 2012;86(1):1-14.

47. Liu Y, Wang DK, Chen LM. The physiology of bicarbonate transporters in mammalian reproduction. Biol Reprod. 2012;86(4):99.

48. Puga Molina LC, Pinto NA, Torres Rodriguez P, Romarowski A, Vicens Sanchez A, Visconti PE, Darszon A, Trevino CL, Buffone MG. Essential role of cftr in pka-dependent phosphorylation, alkalinization, and hyperpolarization during human sperm capacitation. J Cell Physiol. 2017;232(6):1404-14.

49. Hernandez-Gonzalez EO, Trevino CL, Castellano LE, de la Vega-Beltran JL, Ocampo AY, Wertheimer E, Visconti PE, Darszon A. Involvement of cystic fibrosis transmembrane conductance regulator in mouse sperm capacitation. J Biol Chem. 2007;282(33):24397-406.

50. Jose O, Torres-Rodriguez P, Forero-Quintero LS, Chavez JC, De la Vega-Beltran JL, Carta F, Supuran CT, Deitmer JW, Trevino CL. Carbonic anhydrases and their functional differences in human and mouse sperm physiology. Biochem Biophys Res Commun. 2015;468(4):713-8.

51. Brenker C, Goodwin N, Weyand I, Kashikar ND, Naruse M, Krahling M, Muller A, Kaupp UB, Strunker T. The Catsper channel: a polymodal chemosensor in human sperm. EMBO J. 2012;31(7):1654-65.

52. Navarrete FA, Garcia-Vazquez FA, Alvau A, Escoffier J, Krapf D, Sanchez-Cardenas C, Salicioni AM, Darszon A, Visconti PE. Biphasic role of calcium in mouse sperm capacitation signaling pathways. J Cell Physiol. 2015;230(8):1758-69.

53. Esposito G, Jaiswal BS, Xie F, Krajnc-Franken MA, Robben TJ, Strik AM, Kuil C, Philipsen RL, van Duin M, Conti M, Gossen JA. Mice deficient for soluble adenylyl cyclase are infertile because of a severe sperm-motility defect. Proc Natl Acad Sci USA. 2004;101(9):2993-8.

54. Hess KC, Jones BH, Marquez B, Chen Y, Ord TS, Kamenetsky M, Miyamoto C, Zippin JH, Kopf GS, Suarez SS, Levin LR, Williams CJ, Buck J, Moss SB. The "soluble" adenylyl cyclase in sperm mediates multiple signaling events required for fertilization. Dev Cell. 2005;9(2):249-59.

55. Buffone MG, Wertheimer EV, Visconti PE, Krapf D. Central role of soluble adenylyl cyclase and camp in sperm physiology. Biochim Biophys Acta. 2014;1842(12 Pt B):2610-20.

56. Tresguerres M, Parks SK, Salazar E, Levin LR, Goss GG, Buck J. Bicarbonatesensing soluble adenylyl cyclase is an essential sensor for acid/base homeostasis. Proc Natl Acad Sci. 2009;107(1):442-7.

57. Jaiswal BS, Conti M. Calcium regulation of the soluble adenylyl cyclase expressed in mammalian spermatozoa. Proc Natl Acad Sci USA. 2003;100(19):10676-81.

58. Zippin JH, Chen Y, Straub SG, Hess KC, Diaz A, Lee D, Tso P, Holz GG, Sharp GW, Levin LR, Buck J. Co2/hco3(-)- and calcium-regulated soluble adenylyl cyclase as a physiological atp sensor. J Biol Chem. 2013; 288(46):33283-91.

59. Xia J, Reigada D, Mitchell CH, Ren D. Catsper channel-mediated ca2+ entry into mouse sperm triggers a tail-to-head propagation. Biol Reprod. 2007; 77(3):551-9.

60. Seifert R, Flick M, Bonigk W, Alvarez L, Trotschel C, Poetsch A, Muller A, Goodwin N, Pelzer P, Kashikar ND, Kremmer E, Jikeli J, Timmermann B, Kuhl H, Fridman D, Windler F, Kaupp UB, Strunker T. The Catsper channel controls chemosensation in sea urchin sperm. EMBO J. 2015;34(3):379-92.

61. Strunker T, Weyand I, Bonigk W, Van Q, Loogen A, Brown JE, Kashikar N, Hagen V, Krause E, Kaupp UB. A k+-selective cgmp-gated ion channel controls chemosensation of sperm. Nat Cell Biol. 2006;8(10):1149-54.

62. Kobori H, Miyazaki S, Kuwabara Y. Characterization of intracellular ca(2+) increase in response to progesterone and cyclic nucleotides in mouse spermatozoa. Biol Reprod. 2000;63(1):113-20.

63. Wassarman PM, Jovine L, Litscher ES. A profile of fertilization in mammals. Nat Cell Biol. 2001;3(2):E59-64.

64. Miwa N. Protein-carbohydrate interaction between sperm and the eggcoating envelope and its regulation by dicalcin, a xenopus laevis zona pellucida protein-associated protein. Molecules. 2015;20(5):9468-86.

65. Gupta SK, Bansal P, Ganguly A, Bhandari B, Chakrabarti K. Human zona pellucida glycoproteins: Functional relevance during fertilization. J Reprod Immunol. 2009;83(1-2):50-5.

66. Stevens CF. Quantal release of neurotransmitter and long-term potentiation Cell. 1993;72(Suppl):55-63.
67. Florman HM, Arnoult C, Kazam IG, Li C, O'Toole CM. A perspective on the control of mammalian fertilization by egg-activated ion channels in sperm: a tale of two channels. Biol Reprod. 1998;59(1):12-6.

68. Jessell TM, Kandel ER. Synaptic transmission: a bidirectional and selfmodifiable form of cell-cell communication. Cell. 1993;72(Suppl):1-30.

69. Bailey JL, Storey BT. Calcium influx into mouse spermatozoa activated by solubilized mouse zona pellucida, monitored with the calcium fluorescent indicator, fluo-3. Inhibition of the influx by three inhibitors of the zona pellucida induced acrosome reaction: Tyrphostin a48, pertussis toxin, and 3-quinuclidinyl benzilate. Mol Reprod Dev. 1994;39(3):297-308.

70. Florman HM. Sequential focal and global elevations of sperm intracellular ca2+ are initiated by the zona pellucida during acrosomal exocytosis. Dev Biol. 1994;165(1):152-64.

71. Florman HM, Jungnickel MK, Sutton KA. Regulating the acrosome reaction. Int J Dev Biol. 2008:52(5-6):503-10.

72. Florman HM, Tombes RM, First NL, Babcock DF. An adhesion-associated agonist from the zona pellucida activates $\mathrm{g}$ protein-promoted elevations of internal ca2+ and ph that mediate mammalian sperm acrosomal exocytosis. Dev Biol. 1989;135(1):133-46.

73. Miller DJ, Shi X, Burkin H. Molecular basis of mammalian gamete binding. Recent Prog Horm Res. 2002;57:37-73.

74. Jungnickel MK, Marrero H, Birnbaumer L, Lemos JR, Florman HM. Trp2 regulates entry of $\mathrm{ca} 2+$ into mouse sperm triggered by egg zp3. Nat Cell Biol. 2001;3(5):499-502

75. Bjorkgren I, Alvarez L, Blank N, Balbach M, Turunen H, Laajala TD, Toivanen J, Krutskikh A, Wahlberg N, Huhtaniemi I, Poutanen M, Wachten D, Sipila P. Targeted inactivation of the mouse epididymal beta-defensin 41 alters sperm flagellar beat pattern and zona pellucida binding. Mol Cell Endocrinol. 2016:427:143-54

76. Rehfeld A, Dissing S, Skakkebaek NE. Chemical uv filters mimic the effect of progesterone on ca2+ signaling in human sperm cells. Endocrinology. 2016;157(11):4297-308.

77. Baron L, Fara K, Zapata-Carmona H, Zuniga L, Kong M, Signorelli J, Diaz ES, Morales P. Participation of protein kinases and phosphatases in the progesterone-induced acrosome reaction and calcium influx in human spermatozoa. Androl. 2016;4(6):1073-83.

78. Harper CV, Barratt CL, Publicover SJ. Stimulation of human spermatozoa with progesterone gradients to simulate approach to the oocyte. Induction of [ca(2+)](i) oscillations and cyclical transitions in flagellar beating. J Biol Chem. 2004;279(44):46315-25.

79. Smith JF, Syritsyna O, Fellous M, Serres C, Mannowetz N, Kirichok $Y$, Lishko PV. Disruption of the principal, progesterone-activated sperm ca2+ channel in a Catsper2-deficient infertile patient. Proc Natl Acad Sci USA. 2013;110(17):6823-8.

80. Wendler A, Albrecht C, Wehling M. Nongenomic actions of aldosterone and progesterone revisited. Steroids. 2012;77(10):1002-6.

81. Suarez SS. Control of hyperactivation in sperm. Hum Reprod Update. 2008;14(6):647-57.

82. Lishko PV, Botchkina IL, Kirichok Y. Progesterone activates the principal ca2+ channel of human sperm. Nature. 2011;471(7338):387-91.

83. Strunker T, Goodwin N, Brenker C, Kashikar ND, Weyand I, Seifert R, Kaupp UB. The Catsper channel mediates progesterone-induced ca2+ influx in human sperm. Nature. 2011:471(7338):382-6.

84. Xia J, Ren D. The bsa-induced ca2+ influx during sperm capacitation is Catsper channel-dependent. Reprod Biol Endocrinol. 2009;7:119.

85. Avidan N, Tamary H, Dgany O, Cattan D, Pariente A, Thulliez M, Borot N, Moati L, Barthelme A, Shalmon L, Krasnov T, Ben-Asher E, Olender T, Khen M, Yaniv I, Zaizov R, Shalev H, Delaunay J, Fellous M, Lancet D, Beckmann JS. Catsper2, a human autosomal nonsyndromic male infertility gene. Eur J Hum Genet. 2003;11(7):497-502.

86. Avenarius MR, Hildebrand MS, Zhang Y, Meyer NC, Smith LL, Kahrizi K, Najmabadi H, Smith RJ. Human male infertility caused by mutations in the Catsper1 channel protein. Am J Hum Genet. 2009:84(4):505-10.

87. Jin JL, O'Doherty AM, Wang S, Zheng H, Sanders KM, Yan W. Catsper3 and Catsper4 encode two cation channel-like proteins exclusively expressed in the testis. Biol Reprod. 2005;73(6):1235-42.

88. Ren D, Xia J. Calcium signaling through Catsper channels in mammalian fertilization. Physiology. 2010;25(3):165-75.

89. Chung JJ, Navarro B, Krapivinsky G, Krapivinsky L, Clapham DE. A novel gene required for male fertility and functional Catsper channel formation in spermatozoa. Nat Commun. 2011;2:153. 
90. Li H, Ding $X$, Guan $H$, Xiong $C$. Inhibition of human sperm function and mouse fertilization in vitro by an antibody against cation channel of sperm 1 : the contraceptive potential of its transmembrane domains and pore region. Fertil Steril. 2009;92(3):1141-6.

91. Li H, Ding X, Guo C, Guan H, Xiong C. Immunization of male mice with bcell epitopes in transmembrane domains of Catsper1 inhibits fertility. Fertil Steril. 2012;97(2):445-52.

92. Dallinga JW, Moonen EJ, Dumoulin JC, Evers JL, Geraedts JP, Kleinjans JC. Decreased human semen quality and organochlorine compounds in blood. Hum Reprod. 2002;17(8):1973-9.

93. Pant N, Mathur N, Banerjee AK, Srivastava SP, Saxena DK. Correlation of chlorinated pesticides concentration in semen with seminal vesicle and prostatic markers. Reprod Toxicol. 2004;19(2):209-14.

94. Younglai EV, Foster WG, Hughes EG, Trim K, Jarrell JF. Levels of environmental contaminants in human follicular fluid, serum, and seminal plasma of couples undergoing in vitro fertilization. Arch Environ Contam Toxicol. 2002;43(1):121-6.

95. Kumar R, Pant N, Srivastava SP. Chlorinated pesticides and heavy metals in human semen. Int J Androl. 2000;23(3):145-9.

96. Tavares RS, Mansell S, Barratt CLR, Wilson SM, Publicover SJ, Ramalho-Santos J. P,p'-dde activates Catsper and compromises human sperm function at environmentally relevant concentrations. Hum Reprod. 2013;28(12):3167-77.

97. Carlson AE, Burnett LA, del Camino D, Quill TA, Hille B, Chong JA, Moran MM, Babcock DF. Pharmacological targeting of native Catspe channels reveals a required role in maintenance of sperm hyperactivation. PLoS One. 2009;4(8):e6844.

98. Li L, Liu J, Li J, Ye Z. Pharmacological investigation of voltage-dependent ca2+ channels in human ejaculatory sperm in vitro. J Huazhong Univ Sci Technolog Med Sci. 2006;26(5):607-9.

99. Saha L, Bhargava VK, Garg SK, Majumdar S. Effect of nimodipine on male reproductive functions in rats. Indian J Physiol Pharmacol. 2000;44(4):449-55.

100. Mansell SA, Publicover SJ, Barratt CL, Wilson SM. Patch clamp studies of human sperm under physiological ionic conditions reveal three functionally and pharmacologically distinct cation channels. Mol Hum Reprod. 2014;20(5):392-408.

101. Navarro B, Kirichok Y, Clapham DE. Ksper, a ph-sensitive k+ current that controls sperm membrane potential. Proc Natl Acad Sci. 2007;104(18):7688-92.

102. Zeng XH, Yang C, Kim ST, Lingle CJ, Xia XM. Deletion of the slo3 gene abolishes alkalization-activated k+ current in mouse spermatozoa. Proc Natl Acad Sci U S A. 2011;108(14):5879-84.

103. He Y, Zou Q, Li B, Chen H, Du X, Weng S, Luo T, Zeng X. Ketamine inhibits human sperm function by ca(2+)-related mechanism. Biochem Biophys Res Commun. 2016;478(1):501-6.

104. Luo T, Li N, He YQ, Weng SQ, Wang T, Zou QX, Zeng XH. Emodin inhibits human sperm functions by reducing sperm $[\mathrm{ca}(2+)] \mathrm{i}$ and tyrosine phosphorylation. Reprod Toxicol. 2015;51:14-21.

105. Kim DR, Kim HY, Kim HY, Chang MS, Park SK. Trigonellaesemen enhances sperm motility and the expression of the cation sperm channel proteins in mouse testes. Evid Based Complement Alternat Med. 2015;2015:1-8.

106. Park EH, Kim DR, Kim HY, Park SK, Chang MS. Panax Ginseng induces the expression of Catsper genes and sperm hyperactivation. Asian J Androl. 2014;16(6):845-51.

107. Luo T, Zou QX, He YQ, Wang HF, Wang T, Liu M, Chen Y, Wang B. Matrine compromises mouse sperm functions by a $[\mathrm{ca}(2+)] \mathrm{i}$-related mechanism. Reprod Toxicol. 2016;60:69-75.

108. Mata-Rocha M, Alvarado-Cuevas E, Hernandez-Sanchez J, Cerecedo D, Felix R, Hernandez-Reyes A, Tesoro-Cruz E, Oviedo N. Molecular cloning and analysis of the Catsper1 gene promoter. Mol Hum Reprod. 2013;19(5):336-47.

109. Mata-Rocha M, Hernandez-Sanchez J, Guarneros G, de la Chesnaye E, Sanchez-Tusie AA, Trevino CL, Felix R, Oviedo N. The transcription factors sox5 and sox9 regulate Catsper1 gene expression. FEBS Lett 2014;588(18):3352-60

110. Oh MS, Chang MS, Park W, Kim DR, Bae H, Huh Y, Park SK. Yukmijihwangtang protects against cyclophosphamide-induced reproductive toxicity. Reprod Toxicol. 2007;24(3-4):365-70.

111. Kim W, Kim SH, Park SK, Chang MS. Astragalus Membranaceus ameliorates reproductive toxicity induced by cyclophosphamide in male mice. Phytother Res. 2012;26(9):1418-21.
112. Wennemuth G, Westenbroek RE, Xu T, Hille B, Babcock DF. Cav2.2 and cav2. 3 ( $n$ - and r-type) ca2+ channels in depolarization-evoked entry of $\mathrm{ca} 2+$ into mouse sperm. J Biol Chem. 2000;275(28):21210-7.

113. Zou QX, Peng Z, Zhao Q, Chen HY, Cheng YM, Liu Q, He YQ, Weng SQ, Wang HF, Wang T, Zheng LP, Luo T. Diethylstilbestrol activates Catsper and disturbs progesterone actions in human spermatozoa. Hum Reprod. 2017;32(2):290-8.

\section{Submit your next manuscript to BioMed Central and we will help you at every step:}

- We accept pre-submission inquiries

- Our selector tool helps you to find the most relevant journal

- We provide round the clock customer support

- Convenient online submission

- Thorough peer review

- Inclusion in PubMed and all major indexing services

- Maximum visibility for your research

Submit your manuscript at www.biomedcentral.com/submit
C) Biomed Central 